\title{
The effect of shear and bulk viscosities on elliptic flow
}

\author{
G. S. Denicol ${ }^{a}$, T. Kodama ${ }^{b}$ and T. Koide ${ }^{c}$
}

\begin{abstract}
In this work, we examine the effect of shear and bulk viscosities on elliptic flow by taking a realistic parameterization of the shear and bulk viscous coefficients, $\eta$ and $\zeta$, and their respective relaxation times, $\tau_{\pi}$ and $\tau_{\Pi}$. We argue that the behaviors close to ideal fluid observed at RHIC energies may be related to non-trivial temperature dependence of these transport coefficients.

${ }^{a}$ Institute für Theoretische Physik, Johann Wolfgang Goethe-Universität, Max-von-Laue Str. 1, 60438, Frankfurt am Main, Germany

${ }^{b}$ Instituto de Física, Universidade Federal do Rio de Janeiro, C. P. 68528, 21945-970,

Rio de Janeiro, Brasil

${ }^{c}$ Frankfurt Institute for Advanced Studies, Johann Wolfgang Goethe-Universität, Ruth-Moufang Str. 1, 60438, Frankfurt am Main, Germany
\end{abstract}

\section{Introduction}

Dissipative hydrodynamics has been applied to relativistic heavy ion collisions to understand the viscous nature of the quark gluon plasma (QGP), in particular, to explain the experimental data of the collective flow parameter $v_{2}$ by including the effect of shear viscosity [1].

One of the objectives of these attempts is to determine $\eta / s$, where $\eta$ is the shear viscosity coefficient and $s$ is the entropy density, by comparing hydrodynamical calculations of $v_{2}$ with the measured values. However, in addition to the uncertainties on the initial condition for hydrodynamics and the breakdown of the traditional Cooper-Frye freeze-out procedure in dissipative hydrodynamics [2, 3], there are several difficulties in these attempts. One should note that these studies are mainly based on three assumptions; 1) the effects of other irreversible currents, such as the bulk viscous pressure and heat conductivity, are smaller than those of the shear stress tensor, 2) $\eta / s$ does not strongly depend on temperature and 3) the effects of shear viscosity on observables are mostly characterized by one transport coefficient, $\eta / s$.

In this work, we would like to discuss the validity of these assumptions. First, $\eta / s$ is in general a function of temperature. While the exact temperature dependence of this ratio is still unknown, its qualitative behavior is somehow well understood. As was shown in Ref. [4, $\eta / s$ exhibits a complex temperature dependence showing a minimum near the QCD phase transition. Thus, it is not clear that such a temperature dependence can be approximately replaced by a constant value. 
Second, we remark that the effects of the shear stress tensor (or any other irreversible current) cannot be characterized only by $\eta$. This might be the case for a fluid described by the relativistic Navier-Stokes (RNS) equation. However, as was shown in Refs. [5, 6], hydrodynamical equations which violate causality, such as the RNS equations, are unstable and cannot be used for any application. To solve the problem of acausality and instability, we have to introduce (at least) one more transport coefficient called the relaxation time $\tau_{R}$, which represents the retardation effect in the formation of the irreversible current. Then it is natural to expect that the relaxation time should affect the behavior of $v_{2}$ or any other observable.

Because of the relaxation time, a large $\eta$ does not necessarily induce a large shear stress tensor. To illustrate this, let us consider the retardation effect of an arbitrary irreversible current $J$ which is induced by a thermodynamic force $F$. For the sake of simplicity, we consider a constant $F$. Then, the current is given by

$$
J=D F\left(1-e^{-t / \tau_{R}}\right),
$$

where $D$ and $\tau_{R}$ are the transport coefficients for this respective irreversible current. One can see that if $\tau_{R}$ is small enough compared to the typical scale of the hydrodynamic evolution, $J$ is simply proportional to $D F$. However, if $\tau_{R}$ is not small, we have to consider the competition of $D$ and $\tau_{R}$ to quantify the dissipative effects.

As a matter of fact, the time scale of the hydrodynamic evolution in relativistic heavy-ion collisions is of the order of $10 \mathrm{fm}$. On the other hand, as is shown in Ref. [7], the relaxation time of the shear viscosity $\tau_{\pi}$ can also reach values around $10 \mathrm{fm}$. In this sense, we should not ignore the effect of $\tau_{\pi}$ in the analysis of $v_{2}$.

In this paper, we calculate the effect of the shear and bulk viscosities on elliptic flow by taking a realistic parameterization of the shear and bulk viscous coefficients, $\eta$ and $\zeta$, and the corresponding relaxation times, $\tau_{\pi}$ and $\tau_{\Pi}$. We argue that the behaviors close to ideal fluid observed at RHIC energies may be related to non-trivial temperature dependence of these transport coefficients.

\section{Model}

In this work, we will use the memory function method [10, 11], which is one of the formulations of relativistic dissipative hydrodynamics. For a general metric, the conservation of energy and momentum is expressed as

$$
D_{\mu} T^{\mu \nu}=0
$$

where $D_{\mu}$ denotes the covariant derivative. We use the Landau definition of the four-fluid velocity $u^{\mu}$ in which case the energy-momentum tensor is decomposed as $T^{\mu \nu}=\varepsilon u^{\mu} u^{\nu}-(p+\Pi) \Delta^{\mu \nu}+\pi^{\mu \nu}$, where, $\varepsilon, p, \Pi$ and $\pi^{\mu \nu}$ are the energy density, pressure, bulk viscous pressure and shear stress tensor, respectively. The projection operator is defined as usual, $\Delta^{\mu \nu}=g^{\mu \nu}-u^{\mu} u^{\nu}$. 
In the memory function method, any irreversible current $J$ is induced by a thermodynamic force $F$ as

$$
\tau_{R} u^{\mu} D_{\mu} J+\left(1+\tau_{R} D_{\mu} u^{\mu}\right) J=F .
$$

This equation is derived to take into account time retardation effects and the extensivity of hydrodynamic variables. One can regard this equation as the generalized version of the Maxwell-Cattaneo equation. It should be emphasized that the existence of the non linear term $\tau_{R} D_{\mu} u^{\mu}$ is essential to obtain a stable theory as is discussed in Ref. [11].

By applying this equation to the cases of the shear stress tensor and the bulk viscous pressure, we obtain

$$
\begin{array}{ll}
\tau_{\pi} \Delta^{\mu \nu \lambda \rho} u^{\alpha} D_{\alpha} \pi_{\lambda \rho}+\pi^{\mu \nu} & =\eta \sigma^{\mu \nu}-\tau_{\pi} \pi^{\mu \nu} \theta, \\
\tau_{\Pi} u^{\alpha} D_{\alpha} \Pi+\Pi & =-\zeta \theta-\tau_{\Pi} \Pi \theta,
\end{array}
$$

where $\sigma^{\mu \nu}=D^{<\mu} u^{\nu>}$ and $\theta=D_{\mu} u^{\mu}$ are the thermodynamic forces. We use the traditional notation $A^{\langle\mu \nu\rangle}=\Delta^{\mu \nu \alpha \beta} A_{\alpha \beta}$ where $\Delta^{\mu \nu \alpha \beta}=\frac{1}{2}\left(\Delta^{\mu \alpha} \Delta^{\nu \beta}+\Delta^{\mu \beta} \Delta^{\nu \alpha}-\frac{2}{3} \Delta^{\mu \nu} \Delta^{\alpha \beta}\right)$ is the symmetric traceless double projection operator.

The above hydrodynamical equation are solved numerically in three spatial dimensions. We use the Smoothed Particle Hydrodynamics (SPH) method. See Refs. [12, 11] for details. We use approximately $80000 \mathrm{SPH}$ particles with a smoothing parameter $h=0.6 \mathrm{fm}[12]$.

For simplicity, we use a factorized initial energy density profile into its longitudinal and transverse parts, as proposed by Ref. [13. The transverse part is parameterized as in Ref. [2]. The longitudinal part is taken as in Ref. [13], with the choice of parameters $\eta_{\text {Gaus }}=0.8$ and $\eta_{\text {flat }}=4$. The initial value of the irreversible currents will be taken as zero and the impact parameter is always set as $b=7.5 \mathrm{fm}$. We use the equation of state (EoS) introduced in [2]. As for the freeze out, we used the ordinary Cooper-Frye formula [12]. However, we did not consider non-equilibrium corrections to the oneparticle distribution function, because, as was pointed out in Refs. 2, 3], this correction gives rise to unphysical behaviors and is not reliable. However, this will not affect the conclusions of this paper.

As mentioned in the introduction, the temperature dependence of the transport coefficients is very important. The microscopic formula to calculate these quantities is given in Ref. [7]. However, the application of this formula is still limited to leading order perturbative calculations. Fortunately, in this approximation $\eta$ and $\zeta$ become equivalent to the ones calculated in the Green-Kubo-Nakano formalism and we can use these results. In this paper, we use the lattice QCD calculations [8] for the QGP phase and we use the results from Ref. 9] for the hadron phase. These calculations for $\eta / s$ were fitted and the result is shown in Fig. 2(a). We can see that $\eta / s$ shows a minimum around $T \sim 180 \mathrm{MeV}$. For the bulk viscosity coefficient $\zeta / s$, we use the parameterization showed in Ref. [2] with a critical temperature of $T \sim 180 \mathrm{MeV}$.

Similarly, in leading order calculations, the microscopic formula predicts a simple relation for the shear viscosity, $\tau_{\pi}=\eta / P$. For the bulk viscosity, there is still no result 

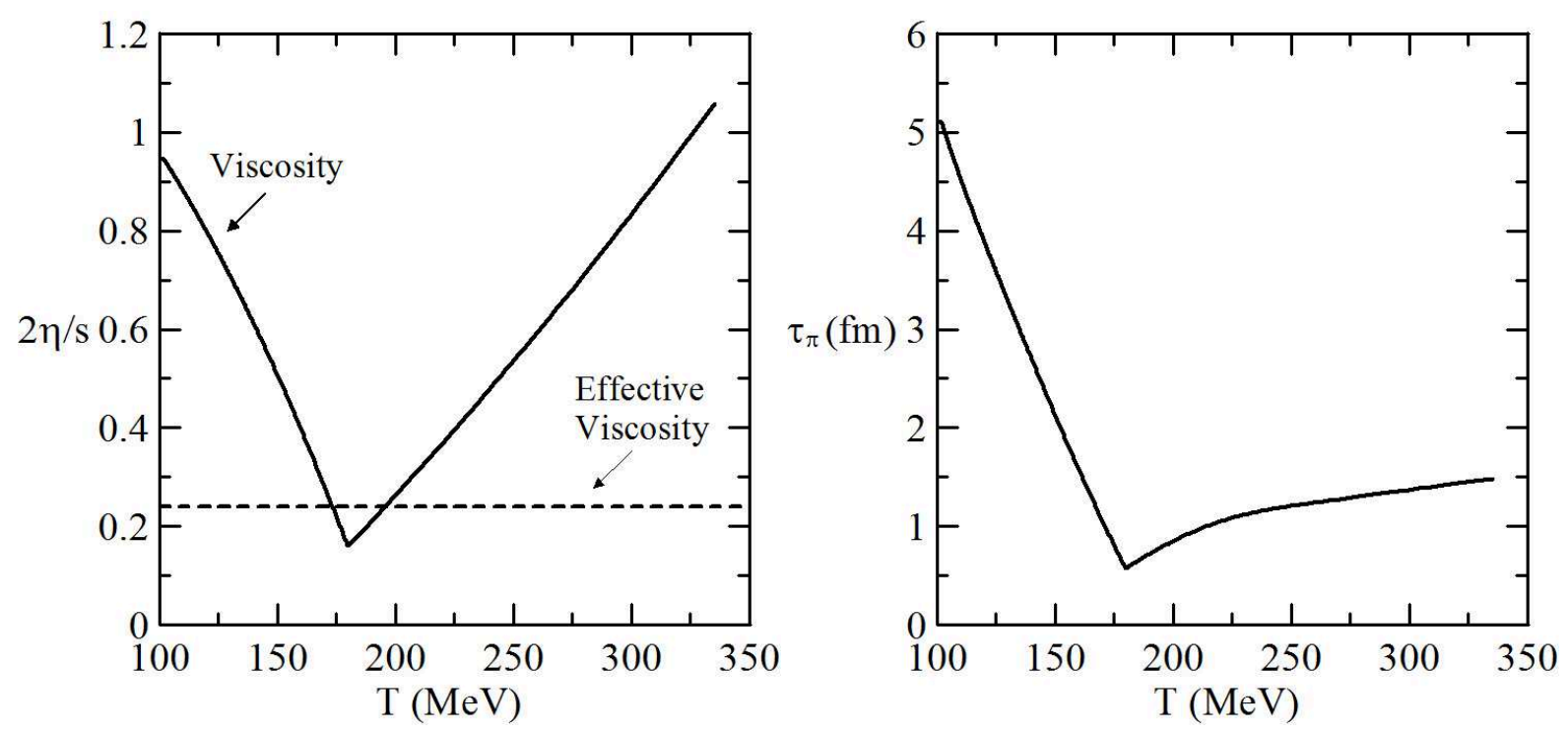

Figure 1. Our parameterization of the shear viscosity $\eta / s$ (left panel) and the shear relaxation time $\tau_{\pi}$ (right panel).
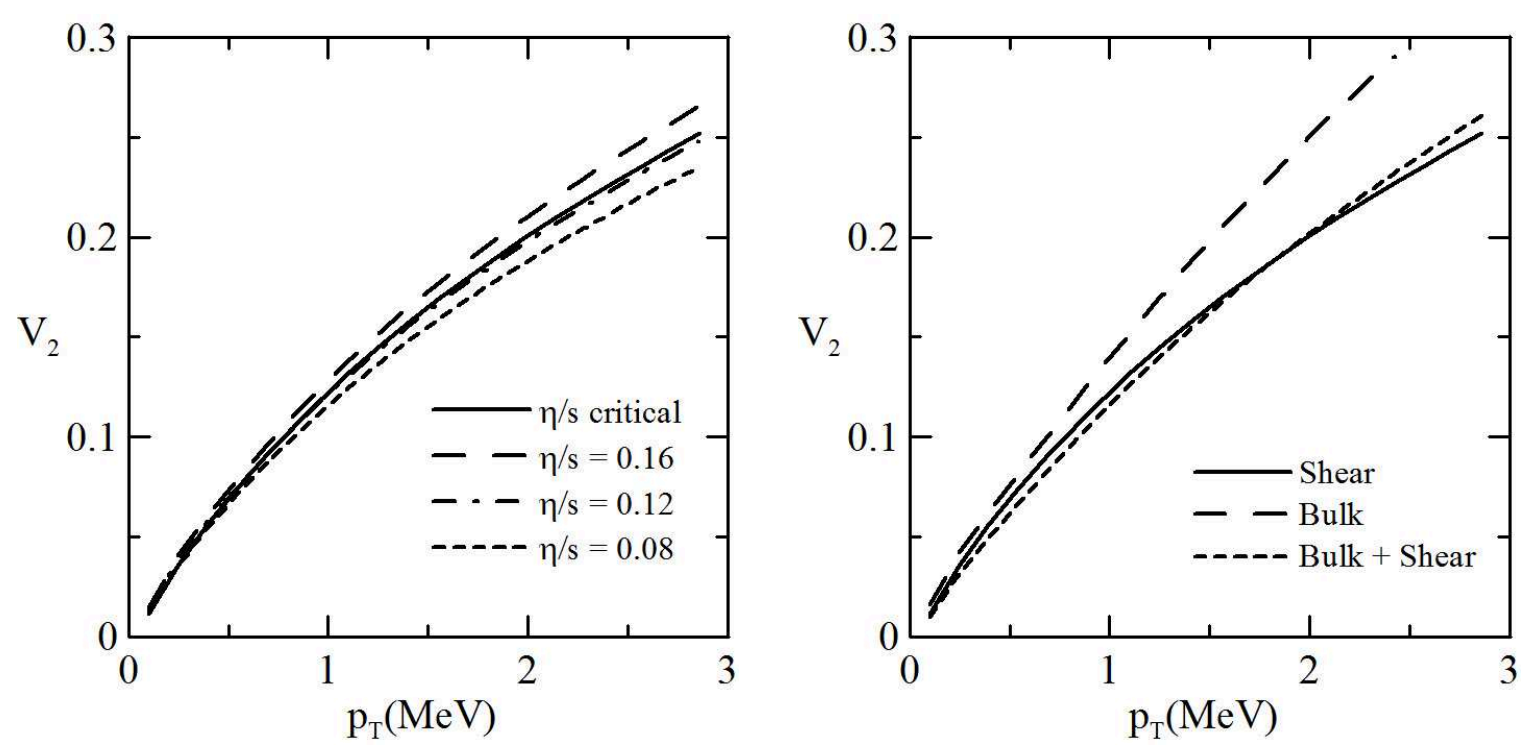

Figure 2. The elliptic flow $v_{2}\left(p_{T}\right)$ calculated for different choices of $\eta / s$ (left panel) and calculated including the effects of bulk viscosity (right panel).

and we simply assume $\tau_{\Pi}=9 \zeta /(\epsilon-3 P)$. Since $\tau_{\pi}\left(\tau_{\Pi}\right)$ is linear with the transport coefficient $\eta(\zeta)$, the critical behavior of $\eta(\zeta)$ is transfered to that of $\tau_{\pi}\left(\tau_{\Pi}\right)$. Thus, $\tau_{\pi}$ will have a minimum around the QCD phase transition while $\tau_{\Pi}$ will display a maximum. In this sense, the complex temperature dependences of the relaxation times are induced by those of $\eta$ and $\zeta$. 


\section{Results}

In Fig. 3(a), we show $v_{2}\left(p_{T}\right)$ for different choices of $\eta / s$ and neglecting bulk viscosity. These calculations were made for thermal pions with a freeze-out temperature of $T_{f}=130 \mathrm{MeV}$. The solid line corresponds to the case where $\eta / s$ has the temperature dependence shown in Fig. 2(a) whereas the dashed, dashed-dotted and dotted lines correspond to constant values of $\eta / s$ of $0.08,0.12$ and 0.16 , respectively. One can see that the elliptic flow calculated with the temperature dependent $\eta / s$ can be well reproduced by the one calculated with an effective constant shear viscosity coefficient $\eta_{\text {eff }} / s=0.12$.

The quantity $\eta_{\text {eff }} / s$ is very close to the minimum of $\eta / s$. This can be explained by the behavior of the relaxation time, shown in Fig. 2(b). As mentioned in the introduction, $\tau_{\pi}$ characterizes the time scale for the velocity gradients to be converted to viscosity. Because the fluid expands with comparable time scale with $\tau_{\pi}$, the magnitude of the shear stress tensor does not become large despite the large values of $\eta / s$ achieved throughout the hydrodynamical evolution.

It should be noted that $\eta_{e f f} / s$ is not bounded by the minimum of $\eta / s$. Let us consider another set of parameters, $\tau_{\pi}=3 \eta / P$. In this case, the effective viscosity $\eta_{\text {eff }} / s$ becomes smaller than the minimum value of $\eta / s$. This result shows that the value of $\eta_{\text {eff }} / s$ is not a direct measurement of $\eta / s$. It can come from the combined effect of $\eta / s$ and $\tau_{\pi}$, and the small values of $\eta_{\text {eff }} / s$ found at RHIC can just as well be a manifestation of the large relaxation time in the hadronic and QGP phases. Whether $\eta_{\text {eff }} / s$ can be used to quantify the value of viscosity at the phase transition or not, depends on the magnitude of the relaxation time in these regions.

In Fig. 3(b), we show $v_{2}\left(p_{T}\right)$ considering the combined effects of bulk and shear viscosities, with realistic transport coefficients. The solid line corresponds to the calculation with only shear viscosity, the dashed line to the calculation with only bulk viscosity and the dashed-dotted line to the calculation with both bulk and shear viscosities. We see that the effect of bulk viscosity is rather small despite the large values of $\zeta / s$ near the phase transition. This can be explained as an effect of the relaxation time $\tau_{\Pi}$, again. Since $\tau_{\Pi}$ also becomes large there, the values of $\zeta / s$ do not generate a large bulk viscous pressure. Since $\zeta$ decreases very quickly outside the phase transition region, we cannot see considerable effects of the bulk viscosity.

We remark that all the calculations showed in this work were done in three spatial dimensions. However, we were mainly interested in $v_{2}$ at zero rapidity, where we confirmed that the Bjorken scaling ansatz is a good approximation. Thus, all the results showed here are approximately equivalent to $(2+1) \mathrm{D}$ hydrodynamical calculations with the Bjorken scaling for the longitudinal direction. This happened mainly because of our choice of initial condition, which displays a plauteu in energy density in the longitudinal direction. Studies with different initial conditions, specially in the longitudinal direction, will be done in future. 


\section{Concluding remarks}

We have shown that due to the interplay between the effects of viscosity and relaxation time, it is possible to fit the behavior of collective flow in terms of one unique effective shear viscosity $\eta_{e f f} / s$. However, its value depends critically on the evolution of the system. Specifically, the value is affected by the time span for which the system remains in different phases. For the LHC energies, for example, we would expect a different value for the effective viscosity since the system will stay longer in the quak gluon plasma phase. However, the details of such changes are hard to calculate due to the uncertainties on $\eta$. We also remark that the uncertainties on the relaxation time are even larger since not so many effort have been applied to calculate the temperature dependence of this transport coefficient (unlike the $\eta / s$ case).

We acknowledge discussions with J. Noronha, P. Huovinen, R. Lacey and D. H. Rischke. This work has been supported by CNPq, FAPERJ, CAPES, PRONEX and the Helmholtz International Center for FAIR within the framework of the LOEWE program (Landesoffensive zur Entwicklung Wissenschaftlich- Okonomischer Exzellenz) launched by the State of Hesse.

[1] See for example, M. Luzum and P. Romatschke, Phys. Rev. C 200878 034915, Erratum-ibid. C 200979 039903, H. Song and U. Heinz, Nucl. Phys. A 2009830 287C-290C and references therein.

[2] G. S. Denicol, T. Kodama, T. Koide and Ph. Mota, Phys. Rev. C 200980064901.

[3] A. Monnai and T. Hirano, Phys. Rev. C 802009054906.

[4] See for example, L. Csernai, J. Kapusta, L. McLerran, Phys. Rev. Lett. 200697152303 and references therein.

[5] G. Denicol, T. Kodama, T. Koide and Philipe Mota, J. Phys. G 200936035103.

[6] S. Pu, T. Koide, D. Rischke, 2009 arXiv:0907.3906 [hep-ph] .

[7] T. Koide, E. Nakano and T. Kodama, Phys. Rev. Lett. 2009103052301.

[8] A. Nakamura and S. Sakai, Phys. Rev. Lett. 2005 94, 072305.

[9] J. Noronha-Hostler, J. Noronha and C. Greiner, Phys. Rev. Lett. 2009103172302.

[10] T. Koide, G. Denicol, Philipe Mota and T. Kodama, Phys. Rev. C 2007 75, 034909.

[11] G. S. Denicol, T. Kodama, T. Koide and Ph. Mota, J. Phys. G 2008 35, 115102.

[12] C. E. Aguiar, T. Kodama, T. Osada e Y. Hama, J. Phys. G 20012775.

[13] T. Hirano, K. Tsuda, Phys. Rev. C 2002 66, 054905. 